\title{
A proposal of a Wireless Sensor Network Routing Protocol
}

\author{
Cláudia Barenco Abbas ${ }^{1}$, Ricardo González ${ }^{1}$, Nelson Cardenas ${ }^{1}$, L. J. García Villalba ${ }^{2}$

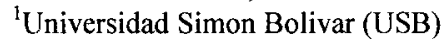 \\ Departamento de Computación y Tecnología de la Información \\ Apartado Postal \\ Caracas, 1080 Venezuela \\ barenco@ldc.usb.ve; rgonzalez@ldc.usb.ve; ncardenas@ldc.usb.ve \\ ${ }^{2}$ Grupo de Análisis, Seguridad y Sistemas (GASS) \\ Departamento de Ingeniería del Software e Inteligencia Artificial-LSI \\ Facultad de Informática, Despacho 431 \\ Universidad Complutense de Madrid (UCM) \\ C/ Profesor José García Santesmases s/n Ciudad Universitaria \\ 28040 - Madrid, Spain \\ javiergv@fdi.ucm.es
}

\begin{abstract}
This paper presents a proposal of a routing protocol to Wireless Sensor Networks, called SHRP (Simple Hierarchical Routing Protocol), whose primary objective is to save battery energy. SHRP also provides both reliability and a load balance solution.

As a novel proposal, it is a proactive protocol that chooses efficient routes, by selecting just the nodes that can contribute to extend the network lifetime. In addition this protocol is also able to inform to a central point about any possible disconnection caused by a reduction of battery power or a long interference period.

The data defined by SHRP protocol can be aggregated during the sending and forwarding tasks. Redundant data are not sent, which contributes to energy saving. This protocol uses the IEEE 802.15.4 under of CC2420 radio chips and it has been implemented on TinyOS operation system.
\end{abstract}

Keywords: 802.15 .4 , routing protocol, wireless sensor network

\section{Introduction}

Monitoring and controlling activities in different systems are fundamental task for improving systems performance. Advances in micro-electronics have allowed, in a great scale, the integration of sensor, microcontroller and communication components in small footprint and low cost devices. These features are making possible the establishment of monitor and control loop in places were it was not possible or affordable until recently.

The sensor node can measure data from any physical system and sent it, usually via radio transmitter, to a command center or sink node, either directly or through a number of 
communication and data concentration devices (or gateways). The decrease in the size and cost of sensors devices has increased the interest in using sets of disposable and unattended sensors. This has led to intensive research addressing with the potential of collaboration among sensors in data collecting, processing and management of the sensing activities, within the last few years.

The main goal of a WSN (Wireless Sensor Networks) is the transmission of data to a base station in an energy efficient. Therefore this networks are concern with lengthening battery life, reducing networks maintenance cost and providing a well connected topology unlike TCP-IP architecture. WSN's has no traditional protocol, because of they limited storage capacity, processing power and battery life, so there is some recent works that try to propose new protocols [1].

\section{Related Works}

Wireless Sensor Network are subject to different requirement depending on the physical environment were they are been instrumented as well as on the network characteristic. The following are some routing protocols for WSN classified according to its network architecture.

\subsection{Flat networks}

In flat networks all network nodes play the same role. The simplest flat protocols are based on either flooding or gossiping mechanism. Flooding involve sending message to any network node, even to destiny by sending data in any direction. This strategy is very simple to implement but it involves sending a lot of messages at the expense of large power consumption, which is not advisable in WSN. The Gossiping strategy consists in sending message, not to all nodes, but to a randomly selected subset of neighbor, this can reduce power consumption however, the arrival of message at lower transmission power cost is not warranted.

SPIN [2] (Sensor Protocol for Information via Negotiation) is designed to send sensed data to any node in the network reducing the amount of data messages. It uses a 3-way handshake with meta-data information to negotiate with each neighbor before transmitting data to them. This avoids the transmission of redundant data in the network.

A node ' $A$ ' intended to transmit the sensed information creates an advertising (ADV) message to send the information to its neighbor. If any neighbor ' $B$ ' wishes to receive the actual data, it sends a request for data message (REQ). Finally ' $A$ ' node sends data messages (DATA) containing actual sensor data to any node $B$ requesting information.

In directed diffusion routing algorithm [3] a human operator's query would be transformed into an interest for named data that is diffused towards nodes in regions $\mathrm{X}$ or $\mathrm{Y}$. Each node 
in the network that forwards this interest message sets up a gradient towards the source of the interest.

A reinforcement process is used to weight the gradients based on their qualities, e.g. loss ratio or hop count. When the sensors report the required information, a message is being returned along the reverse path of interest propagation towards the sink. Intermediate nodes might cache, transform, or aggregate data by combining reports from several sensors, and may direct interests based on previously cached data.

Depending on the number of nodes and the number of active interests, the utilization of the network can be very low comparable to other traditional routing approaches.

AIMRP protocol [4] organizes the network into tiers around a sink, and routes packets by progressively forwarding them to a closer tier from the sink node. This strategy do not requires coordination between the nodes which can repeatedly shut their radio modules off when not in use, independently of the nodes, while satisfying sensor-to-sink latency guarantees.

\subsection{Hierarchical networks}

LEACH [5] (Low-Energy Adaptive Clustering Hierarchy), is a hierarchical protocol built on clusters of nodes that use randomized rotation of local cluster base stations to evenly distribute the energy load among the sensors in the network. Cluster formation is a distributed task based on a subset of node randomly selected as Clusters Head $(\mathrm{CH})$, which aggregate information of its cluster in order to reduce the amount of messages traveling through the network.

In this protocol operation two different phases can be identified: (i) during configuration phase $\mathrm{CH}$ is being elected and cluster being conformed; (ii) in transmission Phase packet is being sent from sensor nodes to a cluster head and then to a Base Station (BS) or sink node. In configuration phase $\mathrm{CH}$ nodes send a broadcast message to other nodes to tell them that it is a new Cluster Head. Based on signal strength each node select to which cluster it's belong to.

TEEN [6] (Threshold sensitive Energy Efficient sensor Network) is other hierarchical protocols for reactive networks, that respond immediately to changes in the relevant parameters. In this protocol a clusters head $(\mathrm{CH})$ sends a hard threshold value and a soft one. The nodes sense their environment continuously. The first time a parameter from the attribute set reaches its hard threshold value, the node switches on its transmitter and sends its data. The nodes then transmits data in the current cluster period if the following conditions are true: the current value of the sensed attribute is greater than the hard threshold, and the current value of the sensed attribute differs from sensed value by an amount equal to or greater than the soft threshold. Both strategy looks to reduce energy spend transmitting messages.

The main drawback of this scheme is that, if the thresholds are not reached, the nodes will never communicate; the user will not get any data from the network at all and will not 
come to know even if all the nodes die. Thus, this scheme is not well suited for applications where the user needs to get data on a regular basis.

DIRq [7] is a protocol that decrease used power by reducing the quantity of messages sent. If a sensor has been registered and sends a value ' $\mathrm{V} 1$ ' for a desired parameter, and in the following measurement period it detect the same value or a similar one, between de (V1$\delta, V 1+\delta$ ) interval then it no do send it again to sink. If sink if not receiving message of a specific node it assume that it sensed value is not been changing too much to be reported.

Flat routing algorithm such as SPIN (Sensor Protocol Information Negotiation) and direct diffusion strategies, seems do not have many application in wireless network sensor of small sizes, and short distances. In a simple network structure in which are not needed many hops to communicate information between sensor nodes, make no sense to use a protocol that make negotiations of complex routes of many hops.

We are interested in considering a protocol that could deal with three different aspects: battery available, number of hops and link quality to guarantee the arrival of messages in the sink node in a energy saving way. We could not find any routing protocol to WSN that use these three parameters together are at the same time be concerned about energy saving and reliability features. By all this reason we propose a new routing protocol.

\section{SHRP Architecture}

SHRP protocol is concerned about topology maintenance that is directed related to the reliability of data delivery. To arrange this it makes use of metrics like local battery available and link quality between neighbor nodes in choosing the best route into the sink node.

Also is concerned about energy saving as not all periodic data is sent to the sink, as is a concern that transmission is the task that more wastes energy in sensor networks [8]. SHRP protocol just sends data that have not changed from the last sensing data. The coordinators nodes can aggregate various data messages and send just one message, in this manner SHRP protocol have also an energy saving behavior.

SHRP provides a load balance scheme during the creation of best routes groups, so no always will be choose the same best route.

To be flexible and to contribute with new metrics for others routing protocols, SHRP architecture uses SP [8] (see figure 1), a specific unifying protocol of TinyOS operating system.

SP allows network protocols to choose neighbors wisely, taking into account information available at the link layer, providing a great modularity.

SHRP protocol periodically monitors the battery lifetime and link quality, cutting off from the routing table nodes that can not contribute in maintaining a well connected topology. 
The proposed protocol takes care about link quality, cutting off neighbor nodes from the routing table nodes that have the average link quality indicator below a minimum threshold. This threshold is related to the IEEE LQI indicator [9].

SHRP protocol cuts off from the routing table also neighbors nodes that have the RSSI (Received Signal Strength Indicator) value below a minimum threshold [10].

Also, SHRP protocol cuts off nodes that do not have battery available, at least to execute what we call "minimum task cycle" [11] (see Equation 1).

$$
\begin{aligned}
& \text { Minimum Task Cycle }=\mathrm{CCA}+\text { Sensing Task }+ \text { Transmission task }+ \text { Reception task }+ \text { Idle } \\
& \text { Period task }
\end{aligned}
$$

All the tasks of cutting off neighbor nodes, showed before, represent that if a node does not have sufficient battery or have a bad link quality, caused by interference, multipath or path loss it will not participate as a route node in the choosing of the best route.

To provide the topology maintenance SHRP protocol defines new metrics that SP does not provide. To be aware about the state of the energy of the nodes, SHRP defines a metric called "minbattrem" that keeps information about the energy available in each node along each route until the sink node.

SHRP protocol tries to choose the route that gives more reliability, this mean, the route that give the mayor energy available along all the possible routes until the sink node.

Another metric defined by SHRP protocol is called "nhops". With this metric SHRP can choose a route that takes into account the number of hops until the sink node. As the transmission is the task that spends more energy, SHRP tries to choose the route that pass through the minor number of hops, saving more energy.

Newer radios that are based on IEEE 802.15.4 standard such as CC2420 implement a parameter called link quality indicator (LQI) which is believed to be a better indicator than RSSI [2]. SHRP protocol uses the metric called "AvgLQI" that represents this parameter.

Protocol designers looking for inexpensive and agile link estimators may choose RSSI over LQI [9], but RSSI at the edge of the threshold of $-87 \mathrm{dBm}$ does not have a good correlation with PRR, so SHRP uses both of the metrics, LQI and RSS, in choosing the best route.

LQI and RSSI metrics are gotten from the link layer of the wireless network. In our experiments we are using the CC2420 radio chip [12].

Figure 1 shows the interaction of SHRP protocol with SP to get information about neighbor nodes and to decide about the best route into the sink node. The metrics shown in bold font are the new ones defined by SHRP protocol. In this manner, other protocol that makes use of any of these metrics will have them already available in SP module. 
SHRP Routing Table

Address - Neighbor Node Identifier

Nhops - Number of Hops until sink node

Minbattrem - Maximum of the minimum of remaining battery

Minavglqi - Maximum of the

minimum of the average LQI

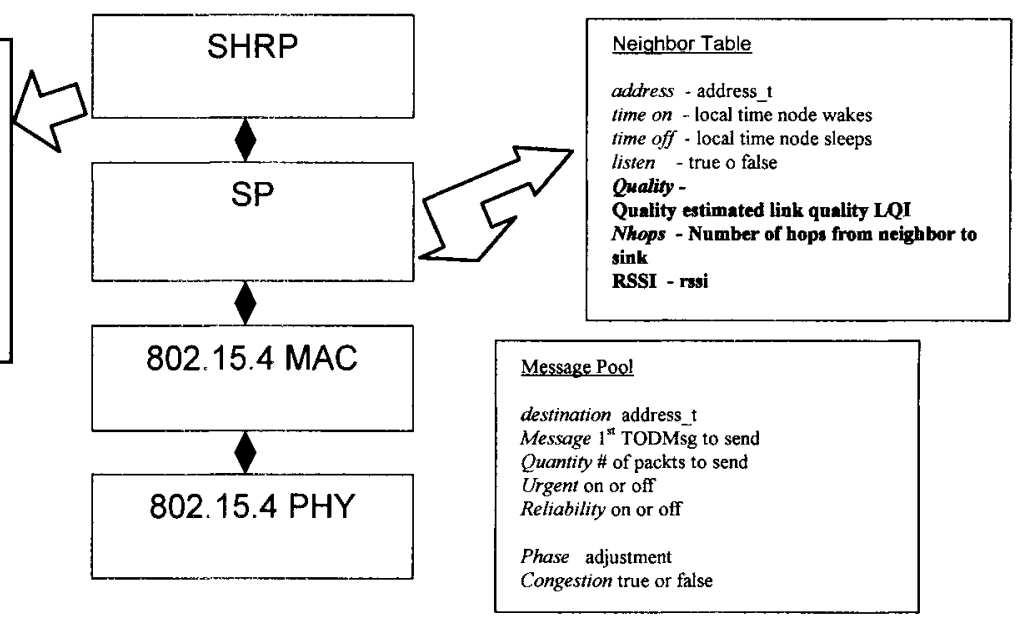

Fig. 1. Interaction between SHRP protocol and SP

SHRP protocol defines three kinds of sensing data messages: (i) periodic; (ii) alert; (iii) alarm [13].

The sink node sends the Query Message, that is explained later, with the information that the monitoring system is interested to collect periodically. Based on this message, the sensing nodes know what and when they have to sense.

The alert message is sent when the sensed data value is above an average value, specified by the monitoring system [13].

The alarm message is sent when the value of the data sensed is below a minimum or above a maximum threshold, also specified by the system [13]. This message is sent with the Urgent and Reliability bits 'on', so they have priority over any other message and the sender waits for an ACK message, to confirm that the alarm message have arrived.

\section{SHRP Functional Description}

There are two different types of nodes defined by SHRP protocol: sensor nodes and coordination nodes.

Sensor Node (SN): This is a node that uses one o more sensors to collect periodically a measure from a physical system, which have to be sent to its coordinator node. Between each period of collecting data the Sensor Node can sleep.

Coordinator Node (CN): This node will have two main functions: (i) route data messages coming from Sensing Node or from another neighbor Coordinator Node; (ii) aggregates messages before sending the received data, in order to decrease transmissions. 


\subsection{Topology Configuration}

SHRP is intended to be used in a sensor network that has a static topology. The static topology can be defined after a site survey process which makes some warranty about network physical connection as any SN should communicate with at least with one CN. Nevertheless, every SN should be associated with only one $\mathrm{CN}$ and statically its know its $\mathrm{CN}$ node.

Each CN can have one or more neighbors, some of them could be reached directly from Sink. Each message sent will arrive to Sink through this CN that should be part of the best route find by SHRP protocol

During network deployment phase some politics could be taken in order to guarantee components redundancies. Even different nodes could be connected to the same sensors (if sensing device is expensive or difficult to find). If we can aggregate additional node to establish redundancy routes, this can impact network topology, increasing network lifetime. This is feasible due to the low cost of motes devices.

Redundant CNs can be used as secondary coordinators (CNsec) that must be not far from its associated primary. Each $\mathrm{CNsec}$ could be in sleep mode and periodically it should check if its primary $\mathrm{CN}$ is alive. If it does no receive any response from $\mathrm{CNsec}$ during a timeout time, it assumes that its primary $\mathrm{CN}$ has gone, so has to do an auto configuration as a primary $\mathrm{CN}$.

\subsection{Messages}

We describe two different types of messages: data and control. Control messages are used to define the tree routes of the SHRP protocol and data messages transmits monitoring system information to the sink node.

\section{Control Messages}

To provide reliability, SHRP protocol uses battery available local $\mathrm{CN}$ information to choose the best route. Others parameter that SHRP uses are RSSI, LQI and number of hops into the sink node. All of them are collected in relation in a peer to peer fashion between neighbor nodes.

Number of hops is calculated based on the Query Message sent periodically by sink node. Further is showed more details about it.

RSSI is an indicator of signal strength and can be used in a simple way to determine if there is a good Reception Packet Rate (PRR). If RSSI is below some threshold, in [10] a

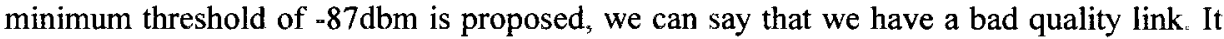
can be seen as a good estimator considering that sensibility radio use to be near of $90 \mathrm{dbm}$. Although RSSI is a metric calculated in the reception of frames, it can be used as a metric 
to the transmission of frames, as new radios chips as CC2420 have an symmetric quality link behavior [10].

LQI is a parameter that comprises the standard IEEE-802.15.4 and is presented in CC2420 radio devices. It represents the error rate in Chips calculated in correlation with the 8 first symbols after SFD (Start Frame 2 Delimiter) [12], so that the value of LQI has a good correlation with the PRR. As LQI changes in time [9], SHRP protocol uses an average of LQI (AvgLQI) calculated within a window frame of a reasonable time.

The principal goal of SHRP protocol is to find a route that increase the network lifetime, that means maintains the connectivity at routing level, and provides a good arrival probability to the Sink Node.

Route selection can not be based only on the data of the next step, since it can lead us to select a way that offers a lower delivery guarantees in later stage. So we propose that some metrics used by the protocol, would be calculated using information from every node that will participate in the selected route.

For example in the battery available metric (battrem), each node must calculate the lower battery available for each one of the possible route to reach Sink Node and choose the greater among them (MaxMinBattAvailable). The same procedure is used with RSSI and AvgLQI metrics, calculating MaxMinRSSI and MaxMinAvgLQI, respectively.

Each CN node sends periodically to neighbor nodes a message called "Network Information Message" (NMI) (see figure 2) which includes updated data of all the metrics mentioned before. As soon as this message is received, $\mathrm{CN}$ node can updated its routing table (see figure 1).

The NMI message format is:

$<$ Address, minAvgLQI, minBattrem, RSSI, AvgLQI>

SHRP protocol keeps in its routing table those nodes that show to be a good neighbor in relation to AvgLQI and RSSI metrics. To verify this feature some thresholds are defined for each metric and every $\mathrm{CN}$ must reach these thresholds to be considered a good neighbor.

Before sending its NMI message, the CN node does an auto evaluation of its battery available metric (battrem). It the value is below a threshold it does not send the NMI message to its neighbor, as it is not a good neighbor node to others nodes. This guarantees that when calculates the best route, $\mathrm{CN}$ node will have just neighbors with a minimum battery available to route messages.

In an intuitive form we can say that a good neighbor is a $\mathrm{CN}$ node that have enough battery to guarantee the shipment of a messages and that offer a reliable way, in terms of radio signal, to reach the Sink node.

The procedures of $\mathrm{CN}$ auto evaluation and the procedure of cutting off the bad neighbor are showed below. 


\begin{tabular}{|} 
If Battrem < BattMin \\
CN does not send NMI message \\
Else For each neighbor node \\
If Neighbor(RSSI)< minRSSI \\
Cutt off the neighbor node as a neighbor \\
Else \\
If Neigbor(LQI) $<$ minLQI \\
Cutt off the neighbor node as a neighbor \\
Endif \\
Endif \\
Mark the neighbor node as a "good" neighbor \\
Endfor
\end{tabular}

Some works have been made to determine reasonable values to be compared against the same metrics used by SHRP. In the case of the available battery, was done a study of battery consumption using $\mathrm{CC} 2420$ radio devices, in order to calculate BattMin level, that a battery should have to send a massage. It can be used to establish some comparisons in terms of energy consumption [11]. In [10] is showed a correlation between average LQI and the rate of reception of packages (PRR). This information could be used to select a reasonable MinLQI that will depend on the lower reception level that the application can reach to guarantee shipment of the package to the next node. If a node decides do not send its NMI, particularly by battery problems, it could send an special message of alert "Control Alarm Message" (CAM) to the sink node, to inform that it will leave the list of neighbors soon, and that this could cause a loose of connectivity in the network.

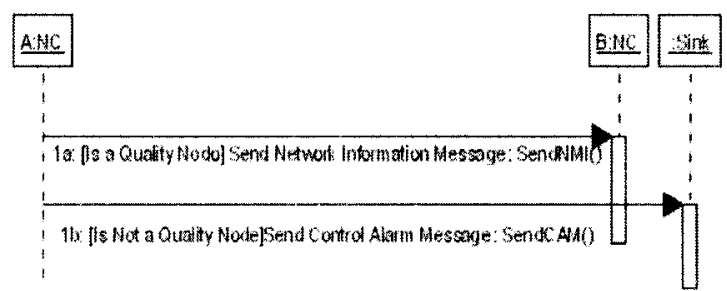

Fig.2: Messages sent to maintain the network topology

After the $\mathrm{CN}$ node has its good neighbor, doing an auto evaluation and cutting off bad neighbors, as explained before, it can start to calculate its best route. Each time it receives a new NMI message, it must calculate the best route. 
Below is represented the algorithm to calculate the best route.

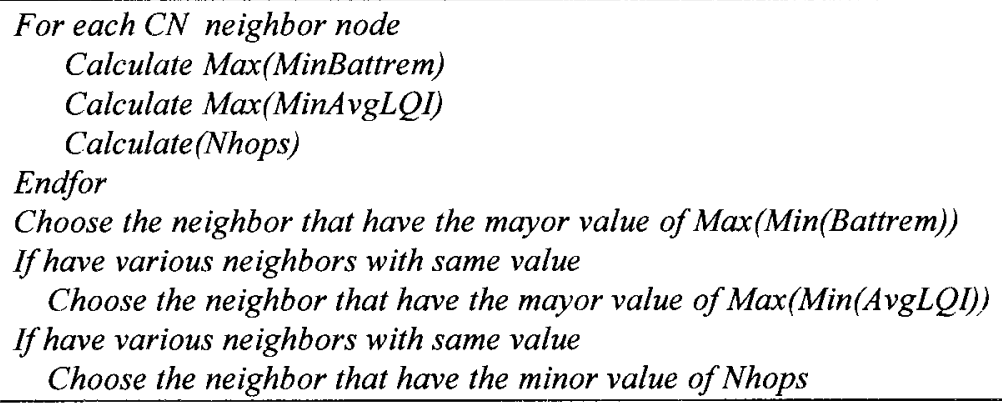

The format of the routing table that uses SHRP can be shown in figure 1 .

\section{Data Messages}

We have three kinds of data messages defined by SHRP protocol: periodically, warnings and alarm.

Lets suppose that a monitoring system needs to obtain information from sensors every time ' $t$ ' and want to be advertised urgently it this value is below a maximum o minimum threshold. SHRP defines a periodically message, Query Message and Query Response Message, to advise sensor nodes about the monitoring system intention.

In SHRP protocol the Query Message has the following structure:

<Address, Variable, Period, Min, Max, Variance, Timestamp>

The Sink node will send these parameters through a Query message, by flooding, which guarantees that it will arrive to every $\mathrm{SN}$ node (see figure 3 ).

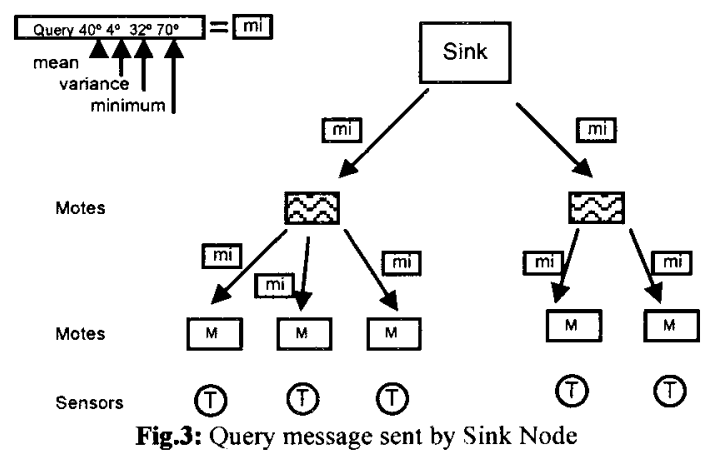


A sequence diagram of the Query Request Message is shown in figure 4.

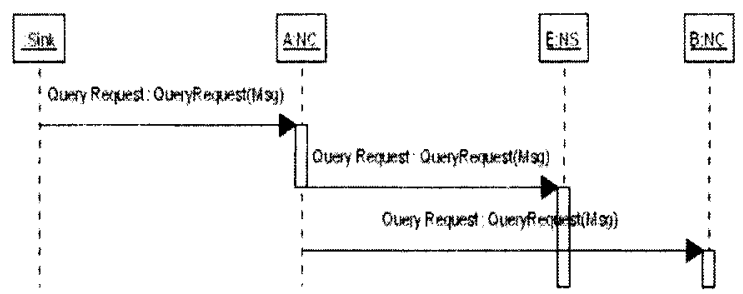

Fig. 4: Query diffusion with parameter that should be measured.

Each node SN based on the regularity of the value to be measured verifies the value sensed and determines if it has changed with respect to the last sensing. If it has changed, send a "Query Response" to its $\mathrm{CN}$, which will be responsible to route the message in direction to the Sink node. If the value sensed keeps constant, the SN node will not send any message to Sink.

As a CN node cannot distinguish between the loss of messages of data of a SN or if the SN is still obtaining the same sensing values, SHRP protocol defines that at each 3 cycles of sensing, SN should send the Query Response message. In this ways the $\mathrm{CN}$ node can detect problems with the $\mathrm{SN}$, and generates an alarm message to the central system.

The coordinator node can aggregate various data of different $\mathrm{SN}$ and send them as a single message reducing the number of transmissions. It can apply this same criterion in case it must route a received message of another $\mathrm{CN}$.

A policy of shipment of obligatory messages each $n$ cycles allows to detect problems and to take actions to correct the connection on the network.

Each SN node must maintain a sensing table for each of its sensors, if it has more than one sensor. The table has the following format:

\section{$<$ Variable, Period, Min, Max, Average, Variance $>$}

Another kind of data messages of SHRP protocol are warning messages. SN node must compare the sensing value with the average of the measured values plus a variance (see equation 2). If the result is greater than the actual sensed value it has to send a warning message. Warning messages are used to inform about some anomaly of the sensed value, but is not a critical situation. Warning messages can be used to keep information about fluctuations in the value measured, which can mean a typical behavior but also can represent that it has some problem.

$$
\text { Last_Sensed_value }>\text { Average+Variance }
$$


If measured values use to have great fluctuations, then a greater variance should be considered in order to avoid constant warning. When a $\mathrm{CN}$ receive a warning it can aggregate it with any message being sent to the Sink node.

The Alarms messages are generated when a measured value exceed a minimum or maximum threshold allowed by the monitoring system (see equation 3 ).

$$
\text { Min }>\text { Last_Sensed_value }>\text { Max }
$$

The Alarm messages with the Reliability and Urgent bits of message pool table of SP configured to ' 1 ' as are urgent messages that must reach the Sink node (see figure 1).

\section{Conclusion}

SHRP protocol uses various quantitative parameters (battery storage, link quality and distance in number of hops up to the sink node) in choosing the best route. These parameter together contribute to have a reliable and energy saving routing protocol. This protocol is a novel proposal as it uses a mix of parameters in choosing the best route.

SHRP protocol enhances the TinyOS operating system as it defines new entries in the SP module neighboring table, so it generates greater modularity.

Also SHRP contributes with the management of the sensor networks nodes, advertising the monitoring system about critical situations of nodes in relation to battery available and link quality.

In the establishing of the link quality metric it not only uses the LQI indicator, but also RSSI indicator, what is a novel usage in the area of routing protocol.

\section{Future Works}

As future work we can mention that we are working in a definition of a network topology auto configuration protocol, using as a principal parameters in the choosing of the $\mathrm{CN}$ node by the SNs nodes, the LQI and RSSI values.

Also we are proposing a reconfiguration protocol for the secondary $\mathrm{CN}$ nodes, in case of the primary $\mathrm{CN}$ is not available any more, so contributing to have a topology fault tolerant protocol. 


\section{References}

1. Al-Karaki, J.N. Kamal, A.E. "Routing Techniques in Wireless Sensor Network: a Survey" Wireless Communications, IEEE Dec. 2004 Volume: 11, Issue: 6. pag 6- 28

2. J. Kulik, W. Heinzelman, and H. Balakrishnan "Adaptive Protocols for Information Dissemination in Wireless Sensor Networks". International Conference on Mobile Computing and Networking archive. Proceedings of the 5th annual ACM/IEEE international conference on Mobile computing and networking. Seattle, Washington.

3. C. Intanagonwiwat, R. Govindan, and D. Estrin, "Directed diffusion: A scalable and robust communication paradigm for sensor networks," in 6th Annual ACM/IEEE International Conference on Mobile Computing and Networking (MobiCOM'00), Boston, MA, USA, August 2000, pp. 56-67.

4. Kulkarni S. , Iyer A and Rosenberg C. "An Address-light, Integrated MAC and Routing Protocol for Wireless Sensor Networks". April 26, 2005.

5. W. R. Heinzelman, A. Chandrakasan, and H. Balakrishnan. "Energy-Efficient Communication Protocol for Wireless Microsensor Networks". In Proc. Of Hawaiian International Conference On Systems Science, January 2000.

6. A. Manjeshwar, D. Agrawal, .'TEEN: A Routing Protocol for Enhanced Efficiency in Wireless Sensor Networks.", in WIRELESS, April 2001.

7. S. Chatterjea, S. De Luigi and P. Havinga, "DirQ: A Directed Query Dissemination Scheme for Wireless Sensor Networks", WSN2006 Wireless Sensor Networks. July, 2006.

8. J. Polastre, J. Hui, P. Levis, J. Zhao, D. Culler, S. Shenker, and I. Stoica, "A unifying link abstraction for wireless sensor networks". In Proceedings of the Third ACM Conference on Embedded Networked Sensor Systems (SenSys 2005).

9. Shan L., Zhang J. et al, "ATPC: Adaptive Transmission Power Control for Wireless Sensor Networks. SenSys'06, November 2006. Bolder, Colorado, USA

10. K.Srinivasan and P. Levis. "RSSI is under appreciated". In Proceedings of the Third ACM Workshop on Embedded Networked Sensors (EmNets 2006), May 2006.

11. Polastre, J., Hill, J., and Culler, D. Versatile low power media access for wireless sensor networks. In Second ACM Conference on Embedded Networked Sensor Systems (2004).

12. CC2420 datasheet, http://www.chipcon.com/files/CC2420 Data Sheet 1 4.pdf

13. Barenco C.; Gonzalez R., Cardenas N., "Proposta de um protocolo de roteamento para Redes Sem Fio de Sensores em Poços de Petróleo". I2TS'2006 - 5th International Information and Telecommunication Technologies Symposium. Cuiaba, Brasil. December 2006. 